\title{
A New Phytoecdysteroid from Ajuga taiwanensis
}

\author{
Yu-Yi CHAN, ${ }^{*, a}$ Tian-Shung Wu, ${ }^{b, c}$ Chang Sheng KUOH, ${ }^{d}$ and Amooru G. DAMU ${ }^{b}$ \\ ${ }^{a}$ Department of Biotechnology, Southern Taiwan University of Technology; Tainan, Taiwan 710, R.O.C.: ${ }^{b}$ Department of \\ Chemistry, National Cheng Kung University; Tainan, Taiwan 701, R.O.C.: ${ }^{c}$ National Research Institute of Chinese \\ Medicine, Taipei; Taiwan 112, R.O.C.: and ${ }^{d}$ Department of Life Sciences, National Cheng Kung University; Tainan, \\ Taiwan 701, R.O.C. Received January 19, 2005; accepted March 26, 2005
}

\begin{abstract}
A new phytoecdysteroid, namely ajugalide-E (1), together with twenty five known compounds were isolated from Ajuga taiwanensis collected from Tainan, Taiwan. Their structures were determined by spectral analysis including high resolution one- and two-dimensional NMR spectroscopy.
\end{abstract}

Key words Ajuga taiwanensis; Labiatae; phytoecdysteroid; ajugalide-E

Many plants contain ecdysteroids, polyhydroxysteroids with a cis-fused $\mathrm{A} / \mathrm{B}$ ring junction and $14 \alpha$-hydroxy-7-ene-6one system, structurally related with ecdysone, the biosynthetic precursor of the insect moulting hormone. ${ }^{1)}$ These compounds exhibit interesting physiological activities such as regulation of moulting in insects, hormonal functions involve regeneration, metamorphosis, reproduction and differentiation in all arthropods, and antiulcer, antirheumatic, insulin regulation and diuretic or tonic effects in mammals. ${ }^{2)}$

Among the numerous plants containing phytoecdysteroids, those pertaining to Ajuga genus are unique for the great variety of such compounds produced. ${ }^{3)}$ Species belonging to genus Ajuga have been used as folk medicinal plants as anthelmintic against intestinal disorders, antifungal, hypoglycaemic, antitumor and antimicrobial agents. ${ }^{4,5}$ Furthermore, it has also been reported that some species of this genus were especially used as diuretic agents, as a remedy for fever, toothache, dysentery and hypertension. ${ }^{6}$ Earlier chemical investigations have led to the isolation of clerodane diterpenoids, ${ }^{7-9)}$ ecdysteroids, ${ }^{3,5,10,11)}$ flavonoids, ${ }^{12)}$ iridoids ${ }^{13-15}$ and triglycerides. ${ }^{16)}$ The compounds isolated from the genus Ajuga, exert a broad spectrum of biological and pharmacological actions such as cytotoxic, anti-mitotic, anti-bacterial, cardiotonic, vasorelaxing, antifungal and antifeedant and insect growth-inhibitions. These facts encouraged us to investigate the chemical constituents of an Ajuga species A. taiwanensis NAKAI ex MuRATA (Labiatae) mainly distributed in the Taiwan, Philippines and Ryukyus which has been often used for the treatment of hepatitis and hepatoma in Taiwanese folk medicine. ${ }^{17)}$ The isolation of ajugalide A to D from title plant has recently been reported from our laboratories. In this paper, we now describe the isolation and structural elucidation of one new phytoecdysteroid, ajugalide-E (1) and twenty five known compounds.

\section{Results and Discussion}

Ajugalide-E (1) was isolated as an optically active, white amorphous solid, $\mathrm{mp} 210-212^{\circ} \mathrm{C}$. The high-resolution fastatom bombardment mass spectrum (HR-FAB-MS) of $\mathbf{1}$ showed a pseudo molecular ion peak $\left([\mathrm{M}+\mathrm{H}]^{+}\right)$at $\mathrm{m} / \mathrm{z}$ 563.3221. This data is combatible with the molecular formula $\mathrm{C}_{31} \mathrm{H}_{47} \mathrm{O}_{9}$, which was also in good agreement with the 31 carbon atom resonances observed in the ${ }^{13} \mathrm{C}$-NMR spectrum. The ${ }^{1} \mathrm{H}-\mathrm{NMR}$ spectra revealed the presence of five methyl groups, two of them doublets with chemical shifts and coupling constants similar to those characteristic ones of phytoecdysteroids with $\gamma$-lactone side chain. ${ }^{18)}$ The occurrence of a $\gamma$-lactone ring in the side chain was evidenced by the carbonyl absorptions at $1730 \mathrm{~cm}^{-1}$ in the IR and $\delta 178.6$ in the ${ }^{13} \mathrm{C}$-NMR spectrum as well as the signal at $\delta 79.2$ assigned to $\mathrm{C}-28$, carbon enclosed in the lactone ring. A low field signal at $\delta 5.43(\mathrm{~d}, J=10.0 \mathrm{~Hz})$ of one deshielded $\mathrm{CH}$ was assigned to H-22 and the adjacent signals of side chain were assigned by the corresponding COSY correlation to H-23 $(2.0-1.6, \mathrm{~m}), \mathrm{H}-24(2.0-1.6, \mathrm{~m}), \mathrm{H}-25$ (2.25, qd, $J=6.8,6.8 \mathrm{~Hz}), \mathrm{Me}-27(1.41, \mathrm{~d}, J=6.8 \mathrm{~Hz}), \mathrm{H}-28(3.95, \mathrm{qd}$, $J=6.8,6.8 \mathrm{~Hz})$ and Me-29 $(1.29, \mathrm{~d}, J=6.8 \mathrm{~Hz})$. These data were consistent with the occurrence of characteristic side chain with $\gamma$-lactone by similitude with 22 -acetylcasterone signals. Additional functionalities included the signal of an olefinic proton at $\delta 6.21(\mathrm{brs})$ as well as nine methine protons and seven methylene groups and an acetyl methyl group also supported the basic skeleton of phytoecdysteroid. As shown in Table 1 the ${ }^{1} \mathrm{H}$ - and ${ }^{13} \mathrm{C}-\mathrm{NMR}$ spectra for compound 1 showed a high analogy with those of 22-acetylcasterone with a cis fused A/B ring junction and $14 \alpha$-hydroxy-7ene-6-one skeleton ${ }^{19)}$ Results of two-dimensional correlation spectroscopy (2D-COSY) and the long-range ${ }^{13} \mathrm{C}-{ }^{1} \mathrm{H}$ correlation in heteronuclear multiple bond correlation (HMBC) experiments also confirmed that the planar structure of $\mathbf{1}$ was the same as 22-acetylcasterone (2). But the differences in the chemical shifts and the multiplicities of A-ring protons suggesting that the new compound might be one epimer at $\mathrm{C}-2$ and/or C-3. The signals of H-2 and H-3 appeared in ${ }^{1} \mathrm{H}-\mathrm{NMR}$ spectrum as ddd with three coupling constants, 7.6, 7.6, $4.0 \mathrm{~Hz}$ characteristic for two diquasiaxial and a quasiaxialquasiequatorial interactions, thereby $\mathrm{H}-2$ and $\mathrm{H}-3$ are in a quasiaxial relative position. These findings also showed the trans relationship of the hydroxyl group at C-2 and C-3. The upfield shifts of $\mathrm{H}-2, \mathrm{H}-1_{\text {ax. }}$ and $\mathrm{H}-5$ revealing the inversion of configuration at $\mathrm{C}-2$, and thus compound $\mathbf{1}$ was a $\mathrm{C}-2$ epimer of $\mathbf{2}$. The final assignment was achieved by NOESY experiment. The presence of NOEs of $\mathrm{H}-5$ with $\mathrm{H}-19$ and H3 with H-9 (Fig. 1) in compounds $\mathbf{1}$ and $\mathbf{2}$ indicated that the $\mathrm{A}$ and $\mathrm{B}$ rings adopted boat-cis-boat conformation. The NOEs between $\mathrm{H}-2$ and $\mathrm{H}-5$ and between $\mathrm{H}-3$ and $\mathrm{H}-9$ showed the hydroxyl groups at $\mathrm{C}-2$ and $\mathrm{C}-3$ to be quasiequatorial, respectively, with trans geometry. On the basis of these results, the structure of ajugalide-E was elucidated as $\mathbf{1}$.

The known compounds, 22-acetylcasterone (2), ${ }^{19)}$ ajugalactone (3) ${ }^{20)}$ cyasterone $(4),{ }^{21)}$ isocyasterone $(5),{ }^{21)}$ ajugamacrin $\mathrm{B}(\mathbf{6}),{ }^{22)}$ ajugapantin $\mathrm{A}(7),{ }^{23)}$ ajugamarin $\mathrm{C} 1(\mathbf{8}),{ }^{15)}$ 
Table 1. ${ }^{1} \mathrm{H}$ - and ${ }^{13} \mathrm{C}$-NMR Spectral Data of Compounds $\mathbf{1}$ and $\mathbf{2}$

\begin{tabular}{|c|c|c|c|c|}
\hline & \multicolumn{2}{|c|}{1} & \multicolumn{2}{|c|}{2} \\
\hline & ${ }^{1} \mathrm{H}-\mathrm{NMR}^{a)}$ & ${ }^{13} \mathrm{C}-\mathrm{NMR}^{b)}$ & ${ }^{1} \mathrm{H}-\mathrm{NMR}^{a)}$ & ${ }^{13} \mathrm{C}-\mathrm{NMR}^{b)}$ \\
\hline \multirow[t]{2}{*}{1} & $2.20-2.00 \mathrm{~m}$ & 33.7 & $2.32-2.12 \mathrm{~m}$ & 38.0 \\
\hline & $2.00-1.60 \mathrm{~m}$ & & $2.04-1.60 \mathrm{~m}$ & \\
\hline 2 & $3.81 \mathrm{ddd}(7.6,7.6,4.0)$ & 75.2 & 4.23 br s & 68.1 \\
\hline 3 & $4.18 \mathrm{ddd}(7.6,7.6,4.0)$ & 71.8 & 4.16 br d (9.2) & 68.1 \\
\hline \multirow[t]{2}{*}{4} & $2.43-2.38 \mathrm{~m}$ & 43.1 & $2.04-1.60 \mathrm{~m}$ & 32.5 \\
\hline & $1.45-1.38 \mathrm{~m}$ & & & \\
\hline 5 & $2.43-2.38 \mathrm{~m}$ & 57.1 & $3.02 \mathrm{dd}(12.8,3.2)$ & 51.4 \\
\hline 6 & & 201.6 & & 203.4 \\
\hline 7 & $6.21 \mathrm{brs}$ & 121.7 & $6.27 \mathrm{~d}(2.0)$ & 122.0 \\
\hline 8 & & 165.6 & & 165.6 \\
\hline 9 & $3.67 \mathrm{t}(9.0)$ & 35.2 & $3.60 \mathrm{t}(8.4)$ & 34.4 \\
\hline 10 & & 39.0 & & 38.8 \\
\hline 11 & $2.00-1.60 \mathrm{~m}$ & 21.0 & $2.04-1.60 \mathrm{~m}$ & 21.1 \\
\hline \multirow[t]{2}{*}{12} & $2.59-2.53 \mathrm{~m}$ & 31.9 & $2.68-2.57 \mathrm{~m}$ & 32.1 \\
\hline & $2.00-1.60 \mathrm{~m}$ & & $2.04-1.60 \mathrm{~m}$ & \\
\hline 13 & & 48.1 & & 48.1 \\
\hline 14 & & 84.0 & & 84.2 \\
\hline \multirow[t]{2}{*}{15} & $2.20-2.00 \mathrm{~m}$ & 31.8 & $2.32-2.12 \mathrm{~m}$ & 31.9 \\
\hline & $2.00-1.60 \mathrm{~m}$ & & $2.04-1.60 \mathrm{~m}$ & \\
\hline \multirow[t]{2}{*}{16} & $2.59-2.53 \mathrm{~m}$ & 21.5 & $2.68-2.57 \mathrm{~m}$ & 21.6 \\
\hline & $2.20-2.00 \mathrm{~m}$ & & $2.32-2.12 \mathrm{~m}$ & \\
\hline 17 & $2.79 \mathrm{t}(8.8)$ & 50.2 & $2.84 \mathrm{t}(9.2)$ & 50.3 \\
\hline 18 & $1.14 \mathrm{~s}$ & 17.8 & $1.19 \mathrm{~s}$ & 17.9 \\
\hline 19 & $1.01 \mathrm{~s}$ & 23.9 & $1.07 \mathrm{~s}$ & 24.5 \\
\hline 20 & & 76.2 & & 76.2 \\
\hline 21 & $1.53 \mathrm{~s}$ & 21.7 & $1.58 \mathrm{~s}$ & 21.8 \\
\hline 22 & $5.43 \mathrm{~d}(10.0)$ & 76.9 & $5.47 \mathrm{~d}(10.4)$ & 76.9 \\
\hline 23 & $2.00-1.60 \mathrm{~m}$ & 33.1 & $2.04-1.60 \mathrm{~m}$ & 33.3 \\
\hline 24 & $2.00-1.60 \mathrm{~m}$ & 48.2 & $2.04-1.60 \mathrm{~m}$ & 48.2 \\
\hline 25 & $2.25 \mathrm{qd}(6.8,6.8)$ & 42.7 & $2.32-2.12 \mathrm{~m}$ & 42.8 \\
\hline 26 & & 178.6 & & 178.7 \\
\hline 27 & $1.41 \mathrm{~d}(6.8)$ & 15.6 & $1.45 \mathrm{~d}(7.2)$ & 15.7 \\
\hline 28 & $3.95 \mathrm{qd}(6.8,6.8)$ & 79.2 & $4.00 \mathrm{qd}(6.4,6.4)$ & 79.3 \\
\hline 29 & $1.29 \mathrm{~d}(6.8)$ & 19.0 & $1.33 \mathrm{~d}(6.4)$ & 19.0 \\
\hline $\mathrm{OH}$ & $6.53 \mathrm{~s}$ & & $6.53 \mathrm{~s}$ & \\
\hline $\mathrm{OH}$ & $5.53 \mathrm{~s}$ & & 6.15 br s & \\
\hline $\mathrm{OH}$ & & & 5.96 br s & \\
\hline $\mathrm{OH}$ & & & $5.64 \mathrm{~s}$ & \\
\hline \multirow[t]{2}{*}{$\mathrm{OAc}$} & $2.01 \mathrm{~s}$ & 170.9 & $2.06 \mathrm{~s}$ & 170.9 \\
\hline & & 21.2 & & 21.3 \\
\hline
\end{tabular}

a) Spectral data were obtained in pyridine- $d_{5}$ at $400 \mathrm{MHz}$. Chemical shift are given in $\delta$ values. b) Spectral data were obtained in pyridine- $d_{5}$ at $100 \mathrm{MHz}$. Chemical shift are given in $\delta$ values.

ajugalide-A (9), ${ }^{24)}$-B $(\mathbf{1 0}),{ }^{24)}-\mathrm{C}(\mathbf{1 1}),{ }^{24)}$-D (12) ${ }^{24)}$ pheophytin-a (13), ${ }^{25)}$ pheophytin-b $(\mathbf{1 4}),{ }^{25)} 13^{2}$-hydroxy $\left(13^{2}-R\right)$ pheophytin-a (15), ${ }^{25)} 13^{2}$-hydroxy $\left(13^{2}-S\right)$ pheophytin-a (16), ${ }^{25)} 8$ $O$-acetylharpagide $(\mathbf{1 7}),{ }^{14)}$ harpagide $(\mathbf{1 8}),{ }^{26)}$ galiridoside (19) ${ }^{14)}$ teuhircoside (20), ${ }^{27)} \beta$-sitosterol (21) ${ }^{28)}$ stigmasterol (22) ${ }^{28)} \beta$-sitosteryl-D-glucoside (23) ${ }^{28)}$ vanillic acid (24) ${ }^{28)}$ kaempferol $(\mathbf{2 5})^{29)}$ and nicotinic acid $(\mathbf{2 6})^{30)}$ were also isolated from Ajuga taiwanensis. Their structures were identified by comparison of their spectroscopic data (UV, IR, NMR and mass spectrometry) with values in the literature.

The chemistry of secondary metabolites of the members of Ajuga including A. taiwanensis appears to be very similar in that all are rich sources of phytoecdysteroids and clerodane diterpenoids, since our phytochemical studies on A. taiwanensis, collected in Taiwan afforded five phytoecdysteroids and seven clerodane diterpenoids. Particularly, the widespread occurrence of various phytoecdysteroids in Ajuga was very significant and their presence might be used as chemotaxonomic marker for the genus.

\section{Experimental}

General Melting points were determined on a Yanagimoto MP-S3 apparatus and are uncorrected. Optical rotations were measured on a JASCO DIP-370 polarimeter. IR and UV spectra were recorded on Shimadzu FTIR8501 and Hitachi UV-3210 spectrophotometers, respectively. EI-MS was obtained on a VG-70-250S mass spectrometer. The ${ }^{1} \mathrm{H}$ - and ${ }^{13} \mathrm{C}-\mathrm{NMR}$, DEPT, COSY, HMQC, NOESY, and HMBC experiments were recorded on a Bruker AMX-400 spectrometer.

Plant Material The whole plant of A. taiwanensis was collected from Tainan, Taiwan, in August 2001 and verified by Prof. C.-S. Kuoh. A voucher specimen (TSWu 90003) has been deposited in the Herbarium of National Cheng Kung University, Tainan, Taiwan.

Extraction and Isolation The dried and powdered whole plants of $A$. taiwanensis $(68 \mathrm{~g})$ was extracted with $\mathrm{MeOH}(\times 10)$ at room temperature and concentrated under reduced pressure to give a dark brown syrup $(14.7 \mathrm{~g})$. The syrup was partitioned succesively between $\mathrm{H}_{2} \mathrm{O}$ and $\mathrm{CHCl}_{3}$. The $\mathrm{CHCl}_{3}$ layer $(3.4 \mathrm{~g}$ ) was chromatographed directly on silica gel and eluted with a gradient of $n$-hexane and EtOAc to afford 13 fractions. Fraction 2 was rechromatographed on silica gel and eluted with $n$-hexane-acetone $(3: 1$, $\mathrm{v} / \mathrm{v})$ to give $\mathbf{1 3}(5 \mathrm{mg}), \mathbf{1 4}(1 \mathrm{mg}), \mathbf{1 5}(0.8 \mathrm{mg}), \mathbf{1 6}(0.7 \mathrm{mg}), \mathbf{2 1}$ and 22 $(3 \mathrm{mg})$. Fractions 6,7 and 8 were combined and rechromatographed on silica gel and eluted with $\mathrm{CHCl}_{3}-\mathrm{MeOH}(30: 1, \mathrm{v} / \mathrm{v})$ to give $6(56 \mathrm{mg})$. Fraction 10 underwent series of chromatographic separations on silica gel using 


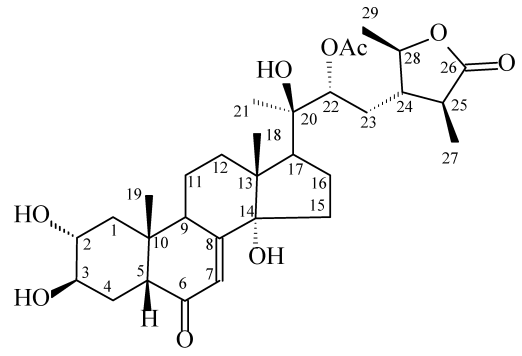

ajugalide-E (1)

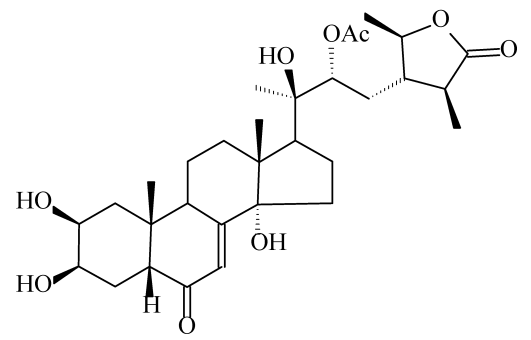

22-acetylcasterone (2)

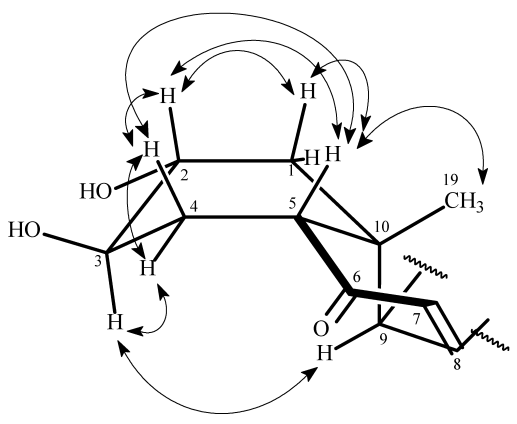

Fig. 1

$\mathrm{CHCl}_{3}-\mathrm{MeOH}(15: 1, \mathrm{v} / \mathrm{v})$ as an eluent to afford $7(8 \mathrm{mg}), 8(11 \mathrm{mg}), 9$ $(2 \mathrm{mg}), \mathbf{1 0}(5 \mathrm{mg}), \mathbf{1 1}(1 \mathrm{mg}), \mathbf{1 2}(0.8 \mathrm{mg})$ and $\mathbf{2 3}(1 \mathrm{mg})$, successively. Fraction 12 was underwent chromatography on silica gel using $\mathrm{CHCl}_{3}-\mathrm{MeOH}$ $(12: 1, \mathrm{v} / \mathrm{v})$ as eluent to afford $\mathbf{1}(2 \mathrm{mg}), \mathbf{2}(2 \mathrm{mg}), \mathbf{3}(1 \mathrm{mg}), \mathbf{4}$ and $\mathbf{5}(1 \mathrm{mg})$ and $26(2 \mathrm{mg})$, respectively. Fraction 13 was also separated by chromatography on silica gel using $\mathrm{CHCl}_{3}-\mathrm{MeOH}(9: 1, \mathrm{v} / \mathrm{v})$ to give $24(0.7 \mathrm{mg})$. The $\mathrm{H}_{2} \mathrm{O}$ layer subjected to column chromatography on Diaion HP-20 and eluted with gradient of $\mathrm{H}_{2} \mathrm{O}-\mathrm{MeOH}$ gave 8 fractions. Fraction 4 was separated by chromatography on silica gel using $\mathrm{CHCl}_{3}-\mathrm{MeOH}(3: 1, \mathrm{v} / \mathrm{v})$ as eluent to give $25(1 \mathrm{mg})$. Fraction 5 was chromatographed on silica gel using a gradient of $\mathrm{CHCl}_{3}-\mathrm{MeOH}(3: 1, \mathrm{v} / \mathrm{v})$ to afford $18(13 \mathrm{mg})$. Fraction 6 was also purified by chromatography on silica gel and eluted with a gradient of $\mathrm{CHCl}_{3}-\mathrm{MeOH}$ to give $20(1 \mathrm{mg})$. Fraction 7 was separated by chromatography on silica gel using $\mathrm{CHCl}_{3}-\mathrm{MeOH}(4: 1, \mathrm{v} / \mathrm{v})$ as eluent to yield 19 $(2 \mathrm{mg})$. Fraction 8 was also separated by chromatography on silica gel using $\mathrm{CHCl}_{3}-\mathrm{MeOH}(5: 1, \mathrm{v} / \mathrm{v})$ as eluent to obtain 17 (96 mg).

Ajugalide-E (1): White amorphous solid, mp $210-212^{\circ} \mathrm{C} .[\alpha]_{\mathrm{D}}^{25}+133.6^{\circ}$ $\left(c=0.007, \mathrm{CHCl}_{3}\right)$. UV $\lambda_{\max }(\mathrm{MeOH}) \mathrm{nm}(\log \varepsilon): 240$ (4.10). IR $(\mathrm{KBr})$ $\mathrm{cm}^{-1}: 3375,1730,1654,1248,1197,1063,1038 .{ }^{1} \mathrm{H}-$ and ${ }^{13} \mathrm{C}-\mathrm{NMR}$ data, see Table 1. FAB-MS $m / z$ : $563\left([\mathrm{M}+\mathrm{H}]^{+}, 1\right), 562(1), 544(1), 526(1), 516$ (1), 508 (3), 498 (2), 484 (1), 466 (1), 456 (1), 451 (1), 448 (2), 433 (2), 377 (2), 371 (13), 363 (3), 360 (2), 353 (3), 345 (2), 327 (3), 301 (4), 300 (3), 299 (3), 250 (3), 249 (5), 183 (8), 149 (100), 139 (20). HR-FAB-MS m/z: 563.3221 (Calcd for $\mathrm{C}_{31} \mathrm{H}_{47} \mathrm{O}_{9}: 563.3220$ ).

22-Acetylcasterone (2): White amorphous solid, mp $212-214^{\circ} \mathrm{C}$. $[\alpha]_{\mathrm{D}}^{25}$ $+111.7^{\circ}\left(c=0.01, \mathrm{CHCl}_{3}\right)$. UV $\lambda_{\max }(\mathrm{MeOH}) \mathrm{nm}(\log \varepsilon): 241$ (4.02). IR $(\mathrm{KBr}) \mathrm{cm}^{-1}: 3382,1769,1735,1655,1259,1188,1042 .{ }^{1} \mathrm{H}-$ and ${ }^{13} \mathrm{C}-\mathrm{NMR}$ data, see Table 1. FAB-MS m/z: $563\left([\mathrm{M}+\mathrm{H}]^{+}, 5\right), 545$ (6), 544 (2), 526 (3),
517 (2), 508 (3), 498 (2), 484 (2), 466 (2), 456 (2), 451 (3), 448 (4), 433 (3), 377 (4), 371 (26), 363 (7), 360 (3), 353 (5), 345 (5), 327 (5), 301 (7), 300 (5), 299 (6), 250 (7), 249 (11), 183 (15), 139 (33), 119 (100). HR-FAB-MS $\mathrm{m} / \mathrm{z}: 563.3218$ (Calcd for $\mathrm{C}_{31} \mathrm{H}_{47} \mathrm{O}_{9}: 563.3220$ ).

Acknowledgments We thank National Science Council, R.O.C. (NSC 90-2113-M-273-001) for financial support of this research.

\section{References}

1) Lafont R., Horn D. H. S., "Ecdysone, from Chemistry to Mode of Action,” ed. by Koolman J., Georg Thieme, Styttgart, 1989, pp. 3964.

2) Rees H. H., "Ecdysone, from Chemistry to Mode of Action," ed. by Koolman J., Georg Thieme, Styttgart, 1989, pp. 28-38.

3) Camps F., Coll J., Phytochemistry, 32, 1361-1370 (1993).

4) Rodriguez-Hann L., Esquivel B., "Cardenas J. Prog. Chem. Org. Nat. Prod.," Springer-Verlag, 1994, p. 63, 107.

5) Wesnner M., Champion B., Girault J. P., Kaouaji N., Saidi B., Lafont R., Phytochemistry, 31, 3785-3788 (1992).

6) Baytop T., "Therapy with Medicinal Plants," (Past and Present) Istanbul University Publications, Istanbul, 1984, pp. 298, 416.

7) Shimomura H., Coll J., Chem. Pharm. Bull., 37, 996-998 (1989).

8) Min Z. D., Wang S. Q., Zheng Q. T., Wu B., Mizuno M., Tanaka T., Iinuma M., Chem. Pharm. Bull., 37, 2505-2508 (1989).

9) Min Z. D., Mizuno M., Wang S. Q., Iinuma M., Tanaka T., Chem. Pharm. Bull., 38, 3167-3168 (1990).

10) Imai S., Toyosato T., Sakai M., Sato Y., Fujioka S., Murata E., Goto M., Chem. Pharm. Bull., 17, 340-342 (1969).

11) Koreeda M., Nakanishi K., Goto M., J. Am. Chem. Soc., 92, 75127513 (1970).

12) Ghedira K., Chemli R., Richard B., Zeches M., Le Men-Olivier L., Plantes. Med. Phytother, 25, 100-111 (1991).

13) Takeda Y., Tsuchida S., Fujita T., Phytochemistry, 26, 2303-2306 (1987).

14) Shimomura H., Sashida Y., Ogawa K., Phytochemistry, 26, 19811983 (1987).

15) Takasaki M., Yamauchi I., Haruna M., Konoshima T., J. Nat. Prod., 61, 1105-1109 (1998).

16) Ben Janet H., Mighri Z., Serani L., Laprevote O., Jullian J. C., Roblot F., Nat. Prod. Lett., 10, 157-164 (1997).

17) Hou D., "Flora of Taiwan," Vol. 4, Editorial Committee of the Flora of Taiwan, Taipei, Taiwan, 1996, p. 442.

18) Calcagno M. P., Camps F., Coll J., Mele E., Sanchezbaeza F., Tetrahedron, 51, 12119-12126 (1995).

19) Saatov Z., Agzamkhodzhaeva D. A., Syrov V. N., Chem. Nat. Comp., 35, 186-191 (1999)

20) Calcagno M. P., Camps F., Coll J., Mele E., Sanchezbaeza F., Tetrahedron, 52, 10137-10146 (1996).

21) Hikino H., Nomoto K., Takemoto T., Chem. Pharm. Bull., 19, 433435 (1971).

22) Shimomura H., Sashida Y., Ogawa K., Chem. Pharm. Bull., 37, 988992 (1989).

23) Shimomura H., Sashida Y., Ogawa K., Chem. Pharm. Bull., 37, 354 357 (1989).

24) Chan Y. Y., Chem. Pharm. Bull., 53 164-167 (2005).

25) Nakatani Y., Ourisson G., Beck J. P., Chem. Pharm. Bull., 29, 22612269 (1981).

26) Sebastien H., Ingrid J. C., Nicolas F., Edouard S., Claude M., Phytochemistry, 51, 767-770 (1999).

27) Jensen H. F. W., Jensen S. R., Nielsen B. J., Phytochemistry, 27, 2581-2589 (1988).

28) Wu T. S., Chan Y. Y., Leu Y. L., Chem. Pharm. Bull., 48, 357-361 (2000).

29) Wu T. S., Leu Y. L., Chan Y. Y., Chem. Pharm. Bull., 46, 1624-1626 (1998).

30) Chen C. Y., Chang F. R., Teng C. M., Wu Y. C., J. Chin. Chem. Soc., 46, 77-86 (1999). 